\title{
Genetic polymorphisms associated with sleep-related phenotypes; relationships with individual nocturnal symptoms of insomnia in the HUNT study
}

\author{
Daniela Bragantini ${ }^{1,2,3^{*}} \mathbb{0}$, Børge Sivertsen ${ }^{2,4,5}$, Philip Gehrman ${ }^{6}$, Stian Lydersen ${ }^{7}$ and Ismail Cüneyt Güzey ${ }^{1,2,3}$
}

\begin{abstract}
Background: In recent years, several GWAS (genome wide association studies) of sleep-related traits have identified a number of SNPs (single nucleotides polymorphism) but their relationships with symptoms of insomnia are not known. The aim of this study was to investigate whether SNPs, previously reported in association with sleep-related phenotypes, are associated with individual symptoms of insomnia.

Methods: We selected participants from the HUNT study (Norway) who reported at least one symptom of insomnia consisting of sleep onset, maintenance or early morning awakening difficulties, (cases, $N=2563$ ) compared to participants who presented no symptoms at all (controls, $N=3665$ ). Cases were further divided in seven subgroups according to different combinations of these three symptoms. We used multinomial logistic regressions to test the association among different patterns of symptoms and 59 SNPs identified in past GWAS studies.

Results: Although 16 SNPS were significantly associated $(p<0.05)$ with at least one symptom subgroup, none of the investigated SNPs remained significant after correction for multiple testing using the false discovery rate (FDR) method.

Conclusions: SNPs associated with sleep-related traits do not replicate on any pattern of insomnia symptoms after multiple tests correction. However, correction in this case may be overly conservative.
\end{abstract}

Keywords: Genetics of insomnia, SNPs, Overlapping phenotypes, Sleep traits, The HUNT study

\section{Background}

In recent years, there has been an increasing focus on the genetic basis for sleep/wake traits. Several genome wide association studies (GWAS) have identified numerous single nucleotide polymorphisms (SNPs) that influence sleep traits [1]. For example, $\mathrm{Hu}$ and colleagues reported 15 SNPs, several of which were on circadian genes, that were significantly associated with being a "morning person" [2] while a study by Gottlieb et al.

\footnotetext{
* Correspondence: daniela.bragantini@ntnu.no

'Department of Research and Development (AFFU), Norwegian University of Science and Technology (NTNU), PO Box 3250 Sluppen, NO-7006 Trondheim, Norway

${ }^{2}$ Department of Mental Health, Norwegian University of Science and Technology (NTNU), PO Box 3250 Sluppen, NO-7006 Trondheim, Norway Full list of author information is available at the end of the article
}

focused on sleep duration identified seven SNPs in two circumscribed genetic loci. Caffeine induced insomnia was the object of another GWAS that identified several loci in melatonin and adenosine pathways [3]. Using the same methodology, Byrne et al. identified several loci with plausible biological role influencing sleep quality and timing [4]. Finally, two studies on the UK biobank sample reported more than a hundred novel SNPs associated to accelerometer registered sleep duration, efficiency and number of nocturnal sleep episodes $[5,6]$ and reproduced three SNPs from previous studies [6].

These sleep-related traits appear to occur with varying frequencies in individuals with insomnia, a condition characterized by decreased quality and/or quantity of sleep in absence of other organic disorders [7]. A study

(c) The Author(s). 2019 Open Access This article is distributed under the terms of the Creative Commons Attribution 4.0 International License (http://creativecommons.org/licenses/by/4.0/), which permits unrestricted use, distribution, and reproduction in any medium, provided you give appropriate credit to the original author(s) and the source, provide a link to the Creative Commons license, and indicate if changes were made. The Creative Commons Public Domain Dedication waiver (http://creativecommons.org/publicdomain/zero/1.0/) applies to the data made available in this article, unless otherwise stated. 
by Vgontzas et al. reported that individuals with short sleep duration are almost five times more likely to suffer from persistent insomnia [8]. Several studies have shown that individuals with evening chronotype are more likely to present insomnia symptoms $[2,9,10]$, in particular difficulties in falling asleep [11]. Therefore, the cooccurrence of specific sleep traits and insomnia might be the product of a common genetic and biological background.

Several GWAS studies seems to support the hypothesis of pleiotropy. A GWAS study by Stein et al. reported an inverted correlation between genetic loci associated with both insomnia and morning chronotype [12]. Several studies conducted on the UK biobank population presented also overlapping genes for insomnia and sleep duration [13-15]. On the other hand, another GWAS study of the same population did not report any common genes for objective measure of several sleep phenotypes [5].

Most studies on insomnia treat insomnia as a single entity and do not consider individual patterns of insomnia symptoms. Insomnia may presents itself as a combination of night-time symptoms but one of these symptom may prevail over the others: difficulties in falling asleep, trouble with staying asleep and waking up too early. Few studies have examined each individual nocturnal symptom of insomnia, despite evidence that the different symptoms may represent biologically distinct mechanisms. Stoffers et al. described decreased gray matter density in a part of the left orbitofrontal cortex in individuals reporting waking up too early, but not in those reporting trouble with falling asleep or sleep maintenance [16]. Epidemiological studies showed that different symptoms are associated with different incidence of physical and psychiatric conditions [17] and mortality [18]. In one such study, males experiencing sleep onset insomnia or terminal insomnia had a risk three-fold higher than healthy sleepers to receive a disability pension due to a mental condition, while maintenance insomnia gave a considerably lower risk.

For these reasons, we argue that investigating individual insomnia symptoms may aid in the identification of genetic overlap with sleep-related traits. Elucidating the relationship between nocturnal insomnia symptoms and sleep-related traits might clarify the etiology and help diagnostic and therapeutic processes.

In order to investigate this relationship, we conducted an association study on individual symptoms of insomnia and SNPs previously reported to be associated with sleep-related phenotypes. The use of material from the Nord-Trøndelag Health Study (HUNT) gave us the opportunity to investigate this relationship in a large sample from a general population.

\section{Methods}

\section{Participants}

This study used data from the Nord-Trøndelag Health Study (HUNT3, Norway) performed in 2006-2008. The study is comprised of 50,807 individuals participated in the study providing extensive health information and biological samples. For a detailed overview of all three HUNT cohorts, see [19].

From the total sample we selected 18,606 participants (36.6\%) who answered "Never/Seldom" to questions about snoring and interrupted breathing during the night (individuals answering "Sometimes" and "Several times a week" were excluded). Of these, participants with complete data for symptoms of insomnia $(N=18,473,99.3 \%)$ were selected. A total of 7933 participants (43\%) could be classified as cases or controls. However, analysis of kinship among these excluded 1262 participants, leaving 6281 participants (79\%). Genetic data was available for 6029 of these participants. The selection workflow is shown in detail in Fig. 1.

\section{Insomnia}

To determine the presence of insomnia symptoms we used three questions contained in the HUNT3 Questionnaire 2 (Sleep section [20]). These three questionnaire items inquire about the frequency of the three core symptoms for insomnia disorder, as specified in the Diagnostic and Statistical Manual of Mental Disorders, Fifth Edition (DSM-5) [7].

"How often in the last 3 months have you:

Had difficulty falling asleep at night?

Woken up repeatedly during the night?

Woken too early and couldn't get back to sleep?”

Possible response options were: "Never/seldom", "Sometimes", "Several times a week".

Answering "Several times a week" to at least one question determined cases $(N=3577)$ while answering "Never/Seldom" to all three questions was used as definition for controls $(N=2452)$.

Cases were further divided in seven subgroups according to the reported pattern of symptoms (Fig. 2).

\section{Genetic data}

We selected the SNPs used in this study the consulting the GWAS catalog [21]. This bioinformatics on-line tool "provides a consistent, searchable, visualizable and freely available database of published SNP-trait associations". In 2016, we searched the GWAS catalog using the keywords "insomnia", "sleep" and "chronotype" and selected 52 SNPs from four GWAS studies [3, 4, 22, 23]. Another 


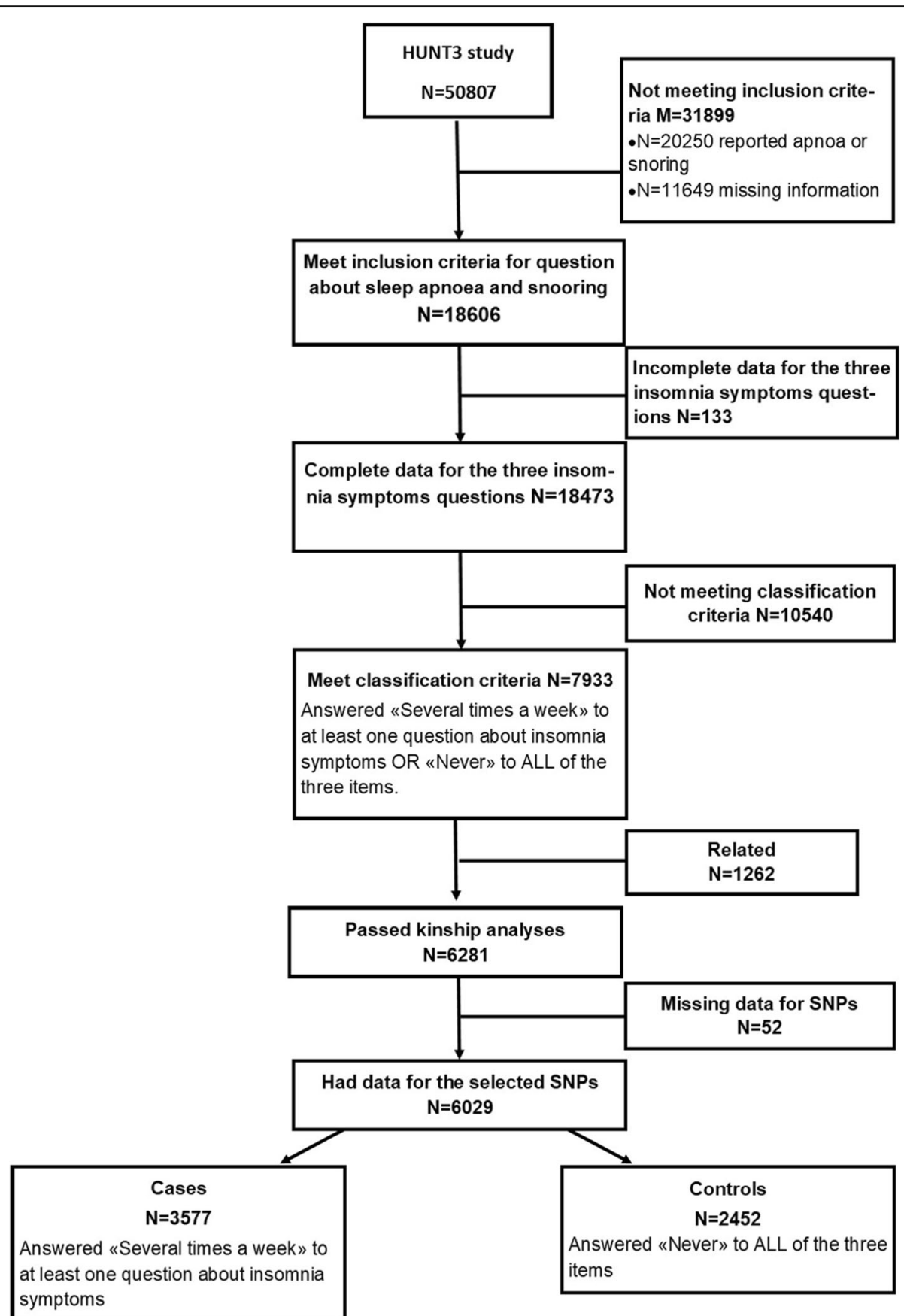

Fig. 1 Selection workflow for the HUNT 3 sample included in this study $(N=6029)$

fifteen SNPs were included from a GWAS study on "morningness" [2] that was not included in the GWAS catalog at the time of the search. A total of 67 SNPs were included in the study.

Genetic data were obtained from the HUNT databank and the genetic material used is stored by the HUNT biobank.
PLINK (version 1.9) [24] was used to exclude SNPs with a minor allele frequency (MAF) below $5 \%$ and those not in Hardy-Weinberg equilibrium ( $p$-value $<0.05)$.

We excluded participants who were related up to the third degree (kinship coefficient $\geq 0.0884$ ) using FastIndep [25]. After kinship analysis, 1262 individuals were excluded (98 cases and 1164 controls). 


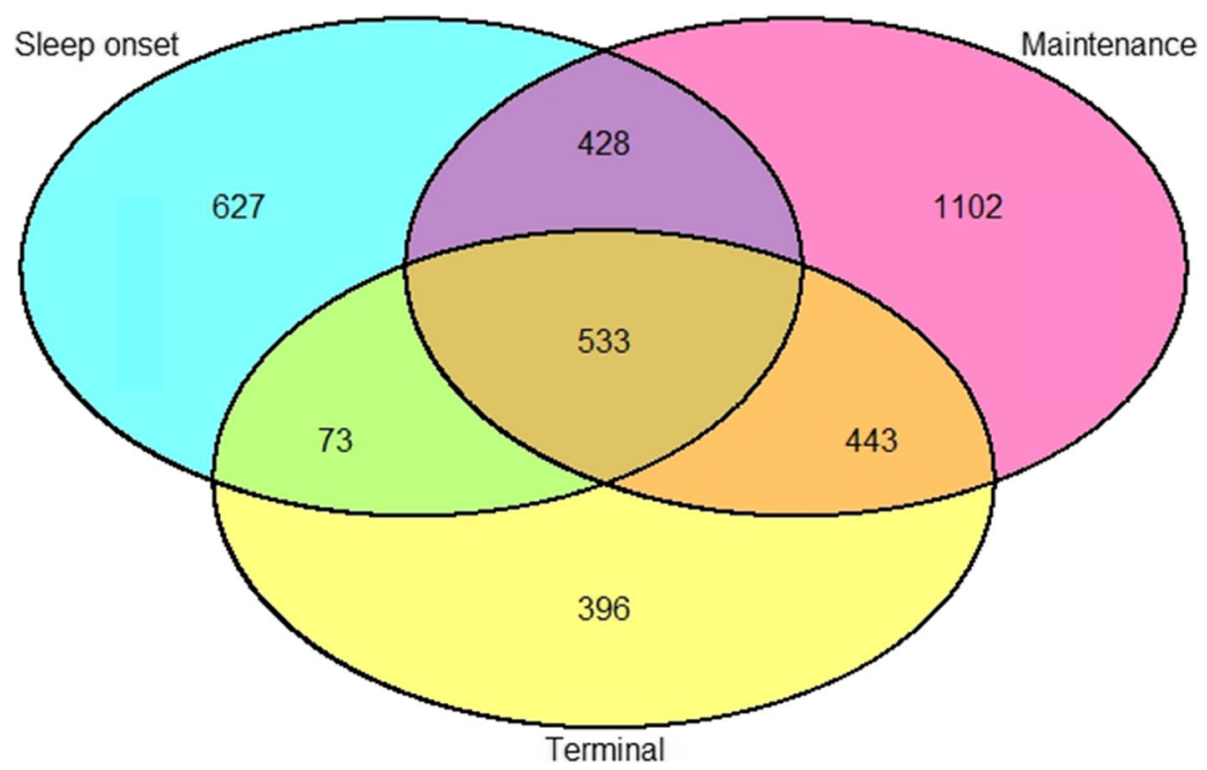

Fig. 2 Distribution of the participants according to reported symptoms of insomnia

\section{Analyses}

We used multinomial logistic regression to test the association between the 67 SNPs and the traits (patterns of symptoms of insomnia plus controls as a dependent variable with eight categories). Sex and age in years were included as covariates. Correction for multiple statistical hypotheses was conducted using Benjamini-Hochberg False Discovery Rate (FDR) (58 SNPs $\times 7$ comparisons for a total of 413 tests, alpha $=0.05)$. All the statistical analyses were conducted using RStudio (Version 1.0.136).

\section{Results}

\section{Descriptive statistics}

Descriptive statistics are shown in Table 1. The current sample $(N=6029)$ included more females $(67 \%)$ than males. Mean age for the whole sample was $50,(\mathrm{SD}=$

Table 1 Descriptive statistics for the sample

\begin{tabular}{llll}
\hline & Count & Females (\%) & Age (M) \\
\hline Sleep onset (SOI) & 627 & 71.1 & 48.9 \\
Maintenance (MI) & 1102 & 72.5 & 53.0 \\
Terminal (TI) & 396 & 60.6 & 57.6 \\
SOI + MI & 428 & 80.6 & 52.1 \\
MI + TI & 443 & 68.6 & 55.6 \\
SOI + TI & 73 & 78.1 & 58.7 \\
SOI + MI + TI & 533 & 83.1 & 55.1 \\
Total in cases & 3602 & $73.1^{*}$ & $53.4^{*}$ \\
Controls & 2427 & 58.2 & 45.2 \\
Total & 6029 & 67.1 & 50.1 \\
\hline
\end{tabular}

${ }^{*}=p<0.05$ for comparison of cases versus controls
16.2, range: 19.2 to 96.8 years), 53 for cases and 45 for controls $(\mathrm{t}(5545,3)=-20.55, p>0.001)$.

\section{Association testing}

Nine SNPs with MAF lower than 5\% in the CEU population (Northern Europeans from Utah) or our sample were excluded. Therefore, a total of 58 SNP were tested for associations.

Sixteen SNPs presented $p$-values below 0.05 before correction for multiple testing in eighteen associations (two SNPs were associated with two symptoms subgroups). None of the p-values retained significance after Benjamini-Hochberg FDR correction.

Besides the p-value, the odds ratio for most of the tests could be considered valid. The T allele of rs2302729 on $C A C N A 1 C$ (Calcium voltage-gated channel subunit alpha1 C) were 1.9 times more likely than controls ( $p=$ $0.004,95 \%$ CI [1.2 to 3$])$ the highest odds ratio for experiencing sleep onset insomnia with terminal insomnia. For the $\mathrm{T}$ allele of rs10493596 on gene AK5 (Adenylate Kinase 5 ) the same symptom was $66 \%$ less likely to occur $(p=0.004, \mathrm{OR}=0.4,95 \% \mathrm{CI}$ [0.3 to 0.8$])$ compared to controls. Individuals who reported sleep onset insomnia with maintenance insomnia were $50 \%$ more likely than controls to present the T allele of rs113851554 on MEIS1 $(p=0.01, \mathrm{OR}=1.5,95 \% \mathrm{CI}[1.1$ to 2$])$. Annotations for all 16 SNPs are collected in Table 2. Results for all SNPs are presented in Additional file 1.

\section{Discussion}

Sixteen SNPs previously associated with sleep-related traits were significantly associated with at least one symptom or a 
Table 2 SNPs showing significant p-value before FDR correction

\begin{tabular}{|c|c|c|c|c|c|c|c|c|c|}
\hline SNP & Ref. allele & Other allele & Gene & $\begin{array}{l}\text { Symptoms } \\
\text { sub-group }\end{array}$ & B & OR & $95 \% \mathrm{Cl}$ & $p$-value & Previous associations \\
\hline rs10493596 & $\mathrm{T}$ & $C$ & AK5 & $\mathrm{SOI}+\mathrm{TI}$ & -0.82 & 0.45 & {$[0.3$ to 0.8$]$} & 0.004 & Morning chronotype [2] \\
\hline rs10823607 & T & $C$ & ADAMTS14 & $\mathrm{SOl}+\mathrm{TI}$ & -0.71 & 0.5 & {$[0.2$ to 0.9$]$} & 0.039 & Sleep duration [4] \\
\hline \multirow[t]{2}{*}{ rs113851554 } & T & G & MEISI & $\mathrm{SOl}+\mathrm{Ml}$ & 0.4 & 1.5 & [1.1 to 2] & 0.007 & Insomnia symptoms [13] \\
\hline & & & & $\mathrm{SOl}+\mathrm{Ml}+\mathrm{Tl}$ & 0.36 & 1.4 & [1.1 to 2] & 0.007 & \\
\hline rs11706236 & G & A & CACNA2D3 & $\mathrm{Ml}+\mathrm{Tl}$ & -0.33 & 0.7 & {$[0.5$ to 0.9$]$} & 0.009 & Caffeine related insomnia [3] \\
\hline rs12471454 & T & C & SATB2 & $\mathrm{Tl}$ & -0.26 & 0.8 & {$[0.6$ to 1$]$} & 0.029 & Insomnia [4] \\
\hline rs12927162 & G & A & TOX3 & $\mathrm{Ml}$ & -0.15 & 0.9 & {$[0.7$ to 1$]$} & 0.036 & Morning chronotype [2] \\
\hline rs1823125 & G & A & PAX8 & $\mathrm{Ml}$ & 0.16 & 1.2 & [1 to 1.4$]$ & 0.033 & Sleep duration [23] \\
\hline rs1940013 & T & $C$ & $O P C M L$ & SOl & 0.19 & 1.2 & [1 to 1.5$]$ & 0.037 & Usual bedtime [22] \\
\hline rs2221285 & T & C & ESRRG & $\mathrm{SOI}+\mathrm{Tl}$ & -0.53 & 0.8 & {$[0.7$ to 1$]$} & 0.027 & Sleep duration [23] \\
\hline rs2287838 & G & A & PIN1 & SOI & -0.22 & 0.8 & {$[0.7$ to 1$]$} & 0.022 & Sleep duration [23] \\
\hline rs2302729 & T & C & CACNATC & $\mathrm{SOI}+\mathrm{Tl}$ & 0.64 & 1.9 & [1.2 to 3$]$ & 0.009 & Sleep latency [4] \\
\hline rs34714364 & T & G & APH1A & Ml & 0.16 & 1.18 & [1 to 1.4$]$ & 0.041 & Morning chronotype [2] \\
\hline rs55694368 & T & G & PER2 & $\mathrm{Ml}$ & -0.19 & 0.83 & {$[0.7$ to 1$]$} & 0.043 & Morning chronotype [2] \\
\hline rs6437122 & G & $C$ & UPP2 & $\mathrm{SOl}+\mathrm{Ml}+\mathrm{Tl}$ & -0.34 & 0.7 & {$[0.5$ to 0.9$]$} & 0.015 & Sleep duration [23] \\
\hline rs9517132 & T & C & RANBP5 & SOI & -0.22 & 0.8 & {$[0.7$ to 1$]$} & 0.018 & Usual sleep duration [23] \\
\hline rs9804200 & C & T & EBF3 & $\mathrm{Ml}+\mathrm{Tl}$ & -0.21 & 0.81 & {$[0.7$ to 1$]$} & 0.044 & Usual bedtime [4] \\
\hline
\end{tabular}

SOI sleep onset insomnia, MI maintenance insomnia, $T I$ terminal insomnia

combination of symptoms of insomnia. However, none of these variations stayed significant after correction for multiple statistical testing.

Among our highest hits, there was SNP rs10493596. This variation is close to the AK5 (Adenylate Kinase 5) gene that encodes for an adenylate kinase expressed exclusively in the brain. This protein is involved in ATP homeostasis by catalyzing the transfer of phosphate groups among adenine nucleotides. Rs10493596 was associated with "morningness" in a study by $\mathrm{Hu}$ et al. [2] while in our study it gave the lowest $p$-value for difficulties falling asleep in combination with early morning awakenings.

Difficulties in falling asleep with early morning awakenings showed also an association with rs2302729 that was previously associated with sleep quality and latency [4]. This SNP is located on CACNA1C (Calcium Voltage-Gated Channel Subunit Alpha1 C) whose involvement in several psychiatric conditions is supported by epidemiological and animal studies [26]. Knockout mice for CACNA1C display traits that resemble symptoms of mental disorders and autism such as cognitive decline, anxiety, hyperactivity, decreased sociability, decreased synaptic plasticity [27].

Of note is the presence of a polymorphism on gene MEIS1 among our highest hits. MEIS1 is involved in restless leg syndrome (RLS) [28] but recently also reported to be associated with insomnia symptoms $[5,13,29]$. In our study, combinations of symptoms sleep onset problems with maintenance insomnia and all symptoms together showed low $p$-values ( 0.01 and 0.02 respectively) and discrete odds ratio (1.5 and 1.4) for the $\mathrm{T}$ allele of rs113851554, in agreement with previous studies. This finding strengthens the hypothesis that insomnia and RLS may be overlapping phenotypes not easy to discern [13].

The A allele of rs12927162 on geneTOX3 (TOX High Mobility Group Box Family Member 3), decreased the chances of maintenance insomnia. This SNP is reported in significant association with being a morning person [2] but also with measure of circadian phase delay [5].

Rs1823125 near gene $P A X 8$ was firstly reported as associated with sleep duration in the CHANGE consortium sample [23], and successively in the UK Biobank sample [6] in which it was associated also with sleep efficiency [5]. In our study, it was associated with maintenance insomnia. PAX8 is a transcription factor with proven role in kidney and thyroid morphogenesis. Its role on sleep is yet to be investigated, and it is possible that rs1823125 is not in fact influencing PAX8 but another gene nearby as it is located in a intragenic region.

Most of our results seems to have a plausible explanation in spite of ending up statistically non-significant after correction for multiple testing. The need for correction when testing multiple hypotheses may often be redundant especially in the context of biology or in K. J. Rothman words “... when scientists are studying biological relations rather than random numbers, the premise that type I errors are the major concern may be wrong" [30]. Therefore overestimating the role of "chance" when analyzing biological data 
may lead to type II errors. In our case, we chose SNPs known to influence sleep-related traits, therefore their involvement in insomnia is plausible.

\section{Strengths and limitations}

The HUNT study collected data from Norwegians from the region of Nord-Trøndelag which gives the advantage of a relative-high genetic isolation and exposure to similar environmental factor that may influence sleep (natural light, cultural habits etc.). Also, strong welfare polices implemented in Norway lessen the effect of socioeconomic disparities that may affect the analyses.

Unfortunately, the HUNT study did not gather information about sleep length or satisfaction that could have helped determining the presence of an actual disorder. Inclusion of this information in future studies and the finding of endophenotypes may help the discovery of relevant genetic associations.

Several of our significant SNPs (before correction for multiple testing) were associated with the subgroup of symptoms "sleep onset with early morning awakenings". This combination of symptoms was the rarest, with only 73 individuals reporting it. This further support the strength of the association.

\section{Conclusions}

After multiple testing correction, we did not find any statistically significant association between combination of symptoms of insomnia and several SNPs associated with sleep-related phenotypes. However, the presence of a biological explanations and early reports on similar phenotypes makes vigorous use of correction for multiple statistical testing questionable.

\section{Supplementary information}

Supplementary information accompanies this paper at https://doi.org/10. 1186/s12881-019-0916-6.

Additional file 1. Full results for the multinomial regression analysis.

\footnotetext{
Abbreviations

ADAMTS14: ADAM Metallopeptidase with Thrombospondin Type 1 Motif 14 AK5: Adenylate Kinase 5; APH1A: Aph-1 Homolog A, Gamma-Secretase Subunit; ATP: Adenosine triphosphate; CACNA1C: Calcium voltage-gated channel subunit alpha1 C; CACNA2D3: Calcium voltage-gated channel auxiliary subunit alpha 2 Delta 3; CEU: Utah Residents (CEPH) with Northern and Western European Ancestry; DSM-5: Diagnostic and Statistical Manual of Mental Disorders, Fifth Edition; EBF3: EBF Transcription Factor 3; ESRRG: Estrogen Related Receptor Gamma; FDR: False discovery rate; GWAS: Genome wide association study; HUNT: Helse Undersøkelse Nørd-Trøndelag (NordTrøndelag Health Study); MAF: Minor allele frequency; MEIS1: Meis Homeobox 1; OPCML: Opioid Binding Protein/Cell Adhesion Molecule Like; PAX8: Paired Box 8; PER2: Period Circadian Regulator 2; PIN1: Peptidylprolyl Cis/Trans Isomerase, NIMA-Interacting 1; RANBP5: Uridine Phosphorylase 2; SATB2: SATB Homeobox 2; SNPS: Single nucleotide polymorphisms; TOX3: TOX High Mobility Group Box Family Member 3; UPP2: Uridine Phosphorylase 2
}

\section{Acknowledgements}

The Nord-Trøndelag Health Study (The HUNT Study) is a collaboration between HUNT Research Centre (Faculty of Medicine, Norwegian University of Science and Technology NTNU), Nord-Trøndelag County Council, Central Norway Health Authority, and the Norwegian Institute of Public Health.

\section{Authors' contributions}

DB: selection, analysis of the genetic data and draft of the manuscript; BS: supervision of the study; PG: critical reading and discussion of manuscript; SL: design and interpretation of the statistical methodology and results; ICG: design and supervision of the study; All authors: critical revision of the manuscript and contribution to the intellectual content of the work. All authors read and approved the final manuscript.

\section{Funding}

This study was funded by the Liaison Committee for education, research and innovation in Central Norway (grant 90061500) and by the Division of Research and Development (AFFU) of the Department of Mental Health, Norwegian University of Science and Technology (NTNU). The funding organs had no role in the design of the study, collection, analysis, and interpretation of data or in writing this manuscript. Dr. Sivertsen had funding from the Norwegian Research Council (project number 239985).

\section{Availability of data and materials}

Data used in this study is available on request to the HUNT databank (https://hunt-db.medisin.ntnu.no/hunt-db/\#/) and is subject to fees as decided by the HUNT Research Centre (https://www.ntnu.edu/hunt/data). The authors of the present study do not have permission to share the dataset obtained from HUNT.

\section{Ethics approval and consent to participate}

This study was approved by the Regional Committee for Medical and Health Research Ethics of South-East Norway (reference number 2016/672) on date 04.27.2016. All participants in the HUNT study signed a written informed consent form allowing the use of their data and samples for research. Participants can demand to have their data deleted from the HUNT database at any given moment.

\section{Consent for publication}

Not applicable.

\section{Competing interests}

The authors declare that they have no competing interests.

\section{Author details}

'Department of Research and Development (AFFU), Norwegian University of Science and Technology (NTNU), PO Box 3250 Sluppen, NO-7006 Trondheim, Norway. ${ }^{2}$ Department of Mental Health, Norwegian University of Science and Technology (NTNU), PO Box 3250 Sluppen, NO-7006 Trondheim, Norway. ${ }^{3}$ St. Olav's University Hospital, Division of Mental Health Care, Østmarkveien 15, NO-7040 Trondheim, Norway. ${ }^{4}$ Department of Health Promotion, Norwegian Institute of Public Health, PO Box 973 Sentrum, 5808 Bergen, Norway. ${ }^{5}$ Department of Research and Innovation, Helse-Fonna HF Haugesund Hospital, PO Box 2170, 5504 Haugesund, Norway. ${ }^{6}$ Department of Psychiatry, University of Pennsylvania Perelman School of Medicine, 3535 Market St., Suite 670, Philadelphia, PA 19104, USA. ${ }^{7}$ Regional Centre for Child and Youth Mental Health and Child Welfare (RKBU), Norwegian University of Science and Technology (NTNU), P.O. Box 8905, N-7491 Trondheim, Norway.

Received: 30 April 2019 Accepted: 31 October 2019

Published online: 12 November 2019

\section{References}

1. Lind MJ, Gehrman PR. Genetic Pathways to Insomnia. Brain Sci. 2016;6(4): 64-81.

2. Hu Y, Shmygelska A, Tran D, Eriksson N, Tung JY, Hinds DA. GWAS of 89,283 individuals identifies genetic variants associated with self-reporting of being a morning person. Nat Commun. 2016;2(7):10448.

3. Byrne EM, Johnson J, McRae AF, Nyholt DR, Medland SE, Gehrman PR, et al. A genome-wide association study of caffeine-related sleep disturbance: 
confirmation of a role for a common variant in the adenosine receptor. Sleep. 2012;35(7):967-75.

4. Byrne EM, Gehrman PR, Medland SE, Nyholt DR, Heath AC, Madden PA, et al. A genome-wide association study of sleep habits and insomnia. Am J Med Genet B Neuropsychiatr Genet. 2013;162b(5):439-51.

5. Jones SE, van Hees VT, Mazzotti DR, Marques-Vidal P, Sabia S, van der Spek A, et al. Genetic studies of accelerometer-based sleep measures yield new insights into human sleep behaviour. Nat Commun. 2019;10(1):1585

6. Dashti HS, Jones SE, Wood AR, Lane JM, van Hees VT, Wang H, et al. Genome-wide association study identifies genetic loci for self-reported habitual sleep duration supported by accelerometer-derived estimates. Nat Commun. 2019;10(1):1100.

7. American Psychiatric Association. Diagnostic and statistical manual of mental disorders (DSM-5๑ ). Arlington, VA, USA: American Psychiatric Pub; 2013.

8. Vgontzas AN, Fernandez-Mendoza J, Bixler EO, Singareddy R, Shaffer ML, Calhoun SL, et al. Persistent insomnia: the role of objective short sleep duration and mental health. Sleep. 2012;35(1):61-8.

9. Suh S, Yang HC, Kim N, Yu JH, Choi S, Yun CH, et al. Chronotype differences in health behaviors and health-related quality of life: a population-based study among aged and older adults. Behav Sleep Med. 2017;15(5):361-76.

10. Chan JW, Lam SP, Li SX, Yu MW, Chan NY, Zhang J, et al. Eveningness and insomnia: independent risk factors of nonremission in major depressive disorder. Sleep. 2014;37(5):911-7.

11. Li SX, Chan NY, Man Yu MW, Lam SP, Zhang J, Yan Chan JW, et al. Eveningness chronotype, insomnia symptoms, and emotional and behavioural problems in adolescents. Sleep Med. 2018;47:93-9.

12. Stein MB, McCarthy MJ, Chen CY, Jain S, Gelernter J, He F, et al. Genomewide analysis of insomnia disorder. Mol Psychiatry. 2018;23(11):2238-50.

13. Lane JM, Liang J, Vlasac I, Anderson SG, Bechtold DA, Bowden J, et al. Genome-wide association analyses of sleep disturbance traits identify new loci and highlight shared genetics with neuropsychiatric and metabolic traits. Nat Genet. 2017:49(2):274-81.

14. Lane JM, Jones SE, Dashti HS, Wood AR, Aragam KG, van Hees VT, et al. Biological and clinical insights from genetics of insomnia symptoms. Nat Genet. 2019;51(3):387-93.

15. Doherty A, Smith-Byrne K, Ferreira T, Holmes MV, Holmes C, Pulit SL, et al. GWAS identifies 14 loci for device-measured physical activity and sleep duration. Nat Commun. 2018;9(1):5257.

16. Stoffers D, Moens S, Benjamins J, van Tol MJ, Penninx BW, Veltman DJ, et al. Orbitofrontal gray matter relates to early morning awakening: a neural correlate of insomnia complaints? Front Neurol. 2012;3:105.

17. Canivet C, Staland-Nyman C, Lindeberg SI, Karasek R, Moghaddassi M, Ostergren PO. Insomnia symptoms, sleep duration, and disability pensions: a prospective study of Swedish workers. Int J Behav Med. 2014;21 (2):319-28.

18. Lallukka T, Podlipskyte A, Sivertsen B, Andruskiene J, Varoneckas G, Lahelma E, et al. Insomnia symptoms and mortality: a register-linked study among women and men from Finland, Norway and Lithuania. J Sleep Res. 2016; 25(1):96-103.

19. Krokstad S, Langhammer A, Hveem K, Holmen TL, Midthjell K, Stene TR, et al. Cohort profile: the HUNT study, Norway. Int J Epidemiol. 2013;42(4):968-77.

20. Engstrøm M, Oslash, Degård S, Sand T, Stovner L, Zwart J, et al. The reliability of a new sleep screening questionnaire for large population-based studies: The third Nord-Trøndelag Health Study. Open Sleep J. 2011;4:14-9.

21. MacArthur J, Bowler E, Cerezo M, Gil L, Hall P, Hastings E, et al. The new NHGRI-EBI catalog of published genome-wide association studies (GWAS catalog). Nucleic Acids Res. 2017;45(Database issue):D896-901.

22. Gottlieb DJ, O'Connor GT, Wilk JB. Genome-wide association of sleep and circadian phenotypes. BMC Med Genet. 2007;8(Suppl 1):S9.

23. Gottlieb DJ, Hek K, Chen TH, Watson NF, Eiriksdottir G, Byrne EM, et al. Novel loci associated with usual sleep duration: the CHARGE consortium genome-wide association study. Mol Psychiatry. 2015;20(10):1232-9.

24. Purcell $S$, Neale B, Todd-Brown $K$, Thomas L, Ferreira MA, Bender D, et al. PLINK: a tool set for whole-genome association and population-based linkage analyses. Am J Hum Genet. 2007;81(3):559-75.

25. Abraham KJ, Diaz C. Identifying large sets of unrelated individuals and unrelated markers. Source Code Biol Med. 2014;9(1):6.

26. Heyes S, Pratt WS, Rees E, Dahimene S, Ferron L, Owen MJ, et al. Genetic disruption of voltage-gated calcium channels in psychiatric and neurological disorders. Prog Neurobiol. 2015;134:36-54.

27. Dedic N, Pohlmann ML, Richter JS, Mehta D, Czamara D, Metzger MW, et al. Cross-disorder risk gene CACNA1C differentially modulates susceptibility to psychiatric disorders during development and adulthood. Mol Psychiatry. 2018:23(3):533-43.

28. Schormair B, Zhao C, Bell S, Tilch E, Salminen AV, Putz B, et al. Identification of novel risk loci for restless legs syndrome in genome-wide association studies in individuals of European ancestry: a meta-analysis. The Lancet Neurol. 2017;16(11):898-907.

29. Hammerschlag AR, Stringer S, de Leeuw CA, Sniekers S, Taskesen E, Watanabe K, et al. Genome-wide association analysis of insomnia complaints identifies risk genes and genetic overlap with psychiatric and metabolic traits. Nat Genet. 2017;49(11):1584-92.

30. Rothman KJ. Six persistent research misconceptions. J Gen Intern Med. 2014;29(7):1060-4.

\section{Publisher's Note}

Springer Nature remains neutral with regard to jurisdictional claims in published maps and institutional affiliations.

\section{Ready to submit your research? Choose BMC and benefit from:}

- fast, convenient online submission

- thorough peer review by experienced researchers in your field

- rapid publication on acceptance

- support for research data, including large and complex data types

- gold Open Access which fosters wider collaboration and increased citations

- maximum visibility for your research: over $100 \mathrm{M}$ website views per year

At BMC, research is always in progress.

Learn more biomedcentral.com/submissions 